\title{
Formación de habilidades investigativas en estudiantes de Cultura Física
}

\section{Training of research skills in Physical Culture students}

\author{
Helder Guillermo Aldas Arcos ${ }^{1 *}$, Carlos Marcelo Ávila Mediavilla ${ }^{1}$ y Yaneisy González Espino ${ }^{2}$ \\ ${ }^{1}$ Universidad Católica de Cuenca, Ecuador \\ 2 Centro de Estudios para el Perfeccionamiento de la Educación Superior, Cuba \\ *hgaldasa@ucacue.edu.ec
}

DOI: https://doi.org/10.26871/killkana_social.v4i1.616

\begin{abstract}
Resumen
En el proceso de formación profesional de los estudiantes de la carrera de Cultura Física a través de un estudio exploratorio se identificó un limitado desarrollo de las habilidades investigativas, las cuales son esenciales para dar respuesta y proponer soluciones a los problemas que se presenten durante su proceso de formación y luego en el ejercicio de la profesión. Para dar respuesta a esta problemática se establecieron los principales sustentos teóricos respecto a la formación de las habilidades investigativas. Se identificaron tres habilidades investigativas esenciales que son: problematizar, teorizar y comprobar la realidad de la profesión. Cada habilidad con sus acciones propician la formación de estas habilidades en los estudiantes, y se implementan desde la cátedra integradora. En este trabajo se presentan acciones de carácter metodológico con el propósito de mejorar la formación de estas habilidades en estudiantes de esta carrera. Para ello, se recomienda la fase de planificación de la tarea docente investigativa que implica la determinación del objetivo, las orientaciones metodológicas organizativas, los contenidos, los métodos, los medios, las formas de organización, de evaluación, el planteamiento de la situación problémica, y las actividades, es decir, las acciones a desarrollar. Luego se sugiere la fase de implementación de tarea docente investigativa, en función de establecer la lógica interna de la actividad investigativa en el proceso docente de la Cátedra Integradora, y se incorporan precisiones que contribuyen al Proyecto Integrador. Este proceso finaliza con la fase de evaluación de la tarea docente investigativa; una de las características de esta tarea es la transversalidad que puede aplicarse semanas de clase o en una unidad.
\end{abstract}

Palabras clave: Habilidades investigativas. Tarea docente investigativa.

\begin{abstract}
In the professional training process of the Physical Culture career students, through an exploratory study, limited development of the investigative skills was identified. These skills are essential for answering and proposing solutions to the problems that arise during their educational process, and later, in their professional practice; in order to address this issue, the main theoretical foundations concerning research skills education were established. Three essential research skills were identified: problematizing, theorizing, and verifying the profession reality. Each skill has its role in the students' development of these skills, and they are implemented from the integrative subject. This work presents methodological approaches aimed to improve the development of these skills in the students of this career. The planning phase of the teaching-research task is recommended. It involves determining the objective, the organizational methodological guidelines, the contents, the methods, the means, the organization, the evaluation, the approach to the problem situation, and the activities, that is to say, the actions to be developed. After that, the research teaching task implementation phase is suggested to establish the internal logic of the research activity in the teaching process of the Integrating Subject. Then, the details that contribute to the Integrating Project are included. The process ends with the evaluation phase of the research teaching task; one of this task's characteristics is the transversality, which can be applied in a unit.
\end{abstract}

Keywords: Research skills. Research teaching task.

\section{Introducción}

En reconocimiento a la importancia de la formación investigativa en Ecuador, la Universidad Católica de Cuenca (UCACUE), aprobó en el año 2016 el Manual de Investigación Formativa, con la finalidad de reglamentar y promover de manera permanente la investigación científica en sus estudiantes (UCACUE, 2016). La finalidad de esta Universidad trata de garantizarse mediante la cátedra integradora, el proyecto integrador y el trabajo de titulación, todos dirigidos a la formación investigativa, no obstante, no es suficiente. 
Con el fin de dar respuesta a esta exigencia, a las demandas sociales, y a las políticas de estado y educativas, la carrera de Ciencias de la Educación mención Cultura Física de la UCACUE tiene como finalidad la formación de un profesional integral. Para ello, se requiere de una constante actualización en teorías, metodologías, técnicas y propuestas contemporáneas, que contribuyan a la solución de los problemas relacionados con la profesión en vínculo con actividades científico investigativas.

En este sentido, la Cultura Física como parte del sistema de influencias de los egresados para la educación de los niños y jóvenes tiene mucho que hacer. Es una disciplina basada en conocimientos de diversas ciencias y por tanto, debe ser científico también el accionar de los profesores, así como el método para identificar y solucionar los problemas relacionados con el desarrollo de la motricidad en niños, el sedentarismo y el sobrepeso que inciden en enfermedades cardiovasculares, además mejorar el modo de vida de la sociedad.

Es por ello, que este trabajo se adentra en el estudio de las habilidades investigativas en la carrera de Cultura Física de la UCACUE, que obtuvo una autoevaluación deficiente, en el indicador participación de estudiantes en las actividades complementarias, relacionadas con eventos científicos, políticos culturales y de investigación de acuerdo al modelo establecido. (CEAACES, 2015)

Así también, las vivencias acumuladas en el ejercicio de la profesión de uno de los autores de este estudio, por la experiencia como: docente, director de esta carrera, subdecano académico, director de trabajos de titulación, además integrante de tribunales de defensa de grado, permitió detectar que los estudiantes al finalizar la carrera muestran insuficiencias en el trabajo de titulación. Para profundizar en esta problemática se realiza un estudio exploratorio en la carrera de Cultura Física de la UCACUE, a partir del análisis documental del perfil de egreso y se hace evidente la necesidad de la formación investigativa en los estudiantes durante esta carrera. Además, en el análisis de los trabajos de titulación presentados en el último ciclo, existen insuficiencias que se reflejan en el diseño teórico metodológico y en la pobre correspondencia entre los resultados teóricos y empíricos.

Sin embargo, el componente investigativo en la formación del profesional de Cultura Física, en sus inicios no estuvo vinculado al perfil de esta carrera y es en la última década, que se reconoce la importancia de la formación investigativa. Esto se refleja en el Diseño Curricular de las universidades ecuatorianas, que como resultado de este proceso exige la presentación de un trabajo de titulación como ejercicio de culminación de la carrera (Larrea, 2013; Aldas, 2014).

A partir de la práctica y el reconocimiento a la importancia del componente investigativo, en la UCACUE se aprueba en mayo del 2016 la concepción del Manual de Investigación Formativa, este documento reglamenta y promueve el proceso investigativo, que comprende la rea- lización de un proyecto integrador para todas las carreras, también en la carrera de Cultura Física.

Este proyecto es el producto de la integración de una o más asignaturas donde se evidencian los contenidos, objetivos y resultados de aprendizaje de los estudiantes dentro del proceso de investigación formativa. Se estructura en dependencia de los niveles establecidos por el Manual, desde el tercero hasta el octavo ciclo de estudio de las carreras. Este proceso lo dirige el profesor de la catedra integradora de cada ciclo de la carrera (UCACUE, 2016).

Esta cátedra constituye una vía para realizar actividades de investigación, con diferentes niveles de complejidad, relacionadas con la carrera correspondiente; así como formar habilidades en los estudiantes, que permitan identificar problemas y necesidades que, potencialmente, se puedan convertir en objeto de estudio.

El Manual, la cátedra integradora y el proyecto integrador; junto a las asignaturas de Metodología de la Investigación y el Trabajo de Titulación constituyen componentes esenciales para la formación investigativa de los estudiantes de la UCACUE. Estos permiten realizar actividades que favorecen la aplicación de los conocimientos científicos y las habilidades investigativas en cada uno de los ciclos de la carrera, lo que representa una exigencia y necesidad actual declarada en las políticas que regulan la educación superior ecuatoriana, que se refleja en la intención expresada en los documentos rectores de la UCACUE.

Por lo que, se sintetiza como dificultad en una relación contradictoria que existe entre la necesidad y exigencias expresadas en el diseño curricular para la formación de las habilidades investigativas en los estudiantes de la carrera de Cultura Física de la UCACUE y las insuficiencias que muestran en la formación de estas. De ahí que, el trabajo tiene como propósito exponer el diseño de acciones para la formación de habilidades investigativas Problematizar, Teorizar y Comprobar en estudiantes de la carrera de Cultura Física, sostenidas en tareas investigativas.

La formación de las habilidades investigativas en la educación superior

Las habilidades investigativas son estudiadas y clasificadas por diferentes autores, Chirino las denomina habilidades científico investigativas, fundamentada en un modelo de enseñanza aprendizaje. Las define como: el "Dominio de las acciones generalizadoras del método científico que potencian al individuo para la problematización, teorización y comprobación de su realidad profesional, lo que contribuye a su transformación sobre bases científicas" (Chirino, 2003, p. 13-14)

Esta autora propone tres importantes habilidades, ellas son: problematizar, teorizar y comprobar, que reflejan una estrecha relación con el proceso investigativo durante la formación profesional, lo que representa una necesidad y exigencia actual en la educación superior.

De acuerdo a la literatura estudiada sobre habilidades investigativas, se asumen las habilidades científico investigativas implementadas por Chirino (2002) y (2005), en la 
realidad educativa, en el contexto de la educación cubana. Para esta investigación se consideran como habilidades investigativas problematizar, teorizar y comprobar la realidad de la Cultura Física, ya que al formarlas el estudiante transita por el proceso investigativo. Esta realidad comprende el ámbito de la Educación Física, el Entrenamiento Deportivo y la Recreación, como áreas de actuación del futuro profesional de esta carrera en Ecuador.

Las habilidades investigativas problematizar, teorizar y comprobar posibilitan la comprensión de la lógica del proceso investigativo, permiten ser formadas desde diferentes espacios del proceso docente y se interrelacionan de manera dialéctica, por lo tanto, para poder contextualizar estas habilidades a la realidad de la Cultura Física, fundamentada en esta teoría, se adecuan las definiciones de la manera siguiente:

Problematizar la realidad de la Cultura Física: es la identificación y determinación de las principales contradicciones en el contexto de actuación del profesional de la Cultura Física en la teoría y práctica, mediante la comparación de lo que acontece en la Educación Física, el Deporte y la Recreación con los conocimientos científicos, lo que permite la identificación de problemas en esta profesión.

El desarrollo de estas contradicciones lleva implícito el análisis desde la teoría científica aplicada a la Cultura Física. Ello permite describir las cualidades, características del objeto de estudio; así como, determinar la situación contradictoria que se presente e identificar el problema profesional.

Teorizar la realidad de la Cultura Física: es la búsqueda, análisis, interiorización y socialización de los conocimientos científicos esenciales para el profesional de la Cultura Física para analizar y fundamentar metodológicamente la realidad profesional con bases teóricas, que den criterios sólidos y permitan asumir posiciones científicas, para llevarlos a la práctica profesional. Exige la utilización de bibliografía confiable, análisis, reflexión para decidir ante diferentes posiciones teóricas, proyectar alternativas de solución y fundamentar los criterios científicos que se asumen, tanto de forma escrita como oral.

Comprobar la realidad de la Cultura Física: es la verificación permanente del proceso y los resultados de la aplicación de las propuestas, que constituyen alternativas científicas de solución a los problemas de la Educación Física, el Deporte y la Recreación, lo que permite valorar sus logros y dificultades desde posiciones científicas, que contribuyan al perfeccionamiento continuo, a partir de su introducción en la práctica de la Cultura Física.

El estudio de la teoría establecida por Chirino, permitió dar cuenta, que para la formación de estas habilidades, es necesario precisar las acciones de cada una. Por ello, esta misma autora propone un conjunto de acciones para cada habilidad, pero fundamenta que estas no son únicas, pues sus acciones dependerán de cada profesión y del contexto en que se desarrolle.

La tarea docente investigativa
En los momentos actuales, en que la formación profesional exige la profundización en el dominio de la investigación científica, la orientación y realización de la tarea docente; así como el enfoque de esta, se considera una de las principales alternativas que puede contribuir al cumplimiento de esta exigencia.

Por ello, la tarea docente es un componente esencial en el proceso docente actual y como cada profesión posee particularidades que la distingue del resto y depende del contexto histórico concreto donde se desempeña y basado en la teoría asumida sobre tarea docente de Álvarez (1996), Chirino (2005), y Sánchez et al. (2010), los autores de este trabajo, definen la tarea docente investigativa; como la célula del proceso formativo, donde el profesor planifica, orienta y controla el proceso contextualizado en la Cultura Física y el estudiante ejecuta de forma productiva y creadora, acciones que le permitan resolver con bases científicas los problemas relacionados con la Educación Física, el Deporte y la Recreación para formar las habilidades problematizar, teorizar y comprobar en cada ciclo de estudio de la carrera de Cultura Física.

Se asume la tarea como célula porque contiene todos los componentes del proceso docente, que incluye al profesor, grupo y al estudiante como sus protagonistas. Esta visión de tarea favorece al profesor para planificar, orientar y controlar las condiciones para el aprendizaje y contribuir a la formación integral del futuro profesional.

Para la implementación del diseño de la tarea, se asumen los rasgos esenciales planteados por Chirino (2005), relacionados con el carácter problematizador, científico e investigativo; el vínculo de la teoría con la práctica y el enfoque profesional.

El diseño de la tarea docente investigativa con carácter teórico práctico en el proceso docente de la carrera de Cultura Física, posibilita que el aprendizaje sea dinámico, activo y productivo. De igual forma, el carácter problematizador científico e investigativo propicia en los estudiantes un espíritu crítico, constructivo y renovador de la realidad, fomenta la búsqueda del conocimiento, el desarrollo del pensamiento científico y la formación de las habilidades investigativas.

En la proyección de la tarea docente para la formación de las habilidades investigativas en los estudiantes de Cultura Física, se tendrán en cuenta el diagnóstico sobre la formación de las habilidades investigativas, la planificación donde se diseña la tarea docente investigativa; así como la ejecución y evaluación como las acciones para formar estas habilidades.

\section{Metodología}

Este estudio se desarrolló con una población 25 estudiantes que conformaron el tercer ciclo de ciclo de la carrera de Ciencias de la Educación Mención Cultura Física, de la Universidad Católica de Cuenca, Sede Azogues.

Los principales métodos utilizados en este estudio fueron los siguientes: 
Histórico-lógico: se utiliza para el análisis cronológico de las concepciones teóricas y metodológicas relacionadas con las habilidades investigativas en estudiantes de Cultura Física.

Analítico-sintético: desde la identificación de la situación problemática, la determinación de los fundamentos teóricos y metodológicos, y la selección de métodos.

Inductivo-deductivo: se utiliza para el estudio de los fundamentos que permiten revelar los aspectos más importantes sobre la formación de las habilidades investigativas en estudiantes de Cultura Física.

Análisis documental: se emplea en la revisión bibliográfica sobre el proceso de formación de las habilidades investigativas en estudiantes de Cultura Física.

\section{Desarrollo y Discusión}

El objetivo es valorar el nivel en que se encuentra la formación de las habilidades investigativas en los estudiantes de la carrera de Cultura Física para la solución de los problemas relacionados con su profesión.

Se toma en cuenta la identificación de los problemas y potencialidades, que inciden en la formación de las habilidades investigativas en los estudiantes de la carrera de Cultura Física, para determinar las condiciones reales que pueden afectar o favorecer la materialización de la misma.

Se debe tener como base el diagnóstico realizado como resultado del análisis del diseño curricular de la carrera, las opiniones recopiladas en los estudiantes y profesores, el tratamiento a las habilidades investigativas en la clase, a los proyectos integradores, que permitieron concebir los problemas y las potencialidades relacionadas con la formación de las habilidades investigativas en el proceso docente de la carrera de Cultura Física. En correspondencia con la concepción de la enseñanza desarrolladora toda acción inicia con un diagnóstico.

\subsection{Fase de Planificación}

El objetivo es diseñar la tarea docente investigativa que contribuyan a la formación de las habilidades investigativas, a partir de los fundamentos teóricos asumidos. Se organizan y proyectan las acciones para la formación de las habilidades investigativas, en los estudiantes de la carrera de Cultura Física. Se realizan las acciones siguientes:

- Establecimiento de la estructura de la tarea docente.

- Desarrollo de la estructura de la tarea docente investigativa, planificación de los tiempos y lugares de ejecución, así como las formas de control.

A partir de los problemas y potencialidades identificados en la etapa de diagnóstico y de los fundamentos teóricos asumidos para la formación de las habilidades investigativas, se determinan el diseño de la Tarea docente investigativa en la cátedra integradora.

\subsubsection{Establecimiento de la estructura de la tarea docente investigativa}

Para el diseño de la tarea docente investigativa se considera la siguiente: Tema: representa el contenido general de la cátedra integradora y/o su derivación en unidades específicas.

Objetivo: Plantear la acción concreta que va a realizar el estudiante en correspondencia con las operaciones que forman la habilidad y con el ejercicio de la profesión. Deben ser expresados en términos de acciones más generales y teniendo en cuenta la función que desempeña la cátedra integradora en la formación del perfil profesional. De ellos derivar las tareas con las cuales se organiza el aprendizaje, precisando las acciones a realizar por el estudiante, el contenido que se va a asimilar en función de los temas a investigar y del desarrollo de habilidades investigativas, así como las condiciones en que se van a desarrollar dichas tareas.

Orientaciones metodológicas organizativas: se explica a los estudiantes como desarrollar la tarea docente investigativa, como irán cumpliendo la tarea durante el desarrollo de los contenidos de la unidad; tiempo a emplear en la tarea dentro o fuera de la clase, formas de organización, métodos, medios, las formas de evaluación y la bibliografía a utilizar en la ejecución de las actividades.

Los contenidos deben ser estructurados interrelacionando todos sus componentes: conocimientos, habilidades y modos de comportamiento, el vínculo con la profesión, en particular los relacionados con la actividad científica.

Los métodos, seleccionados en correspondencia con los objetivos, contenidos de la unidad y las habilidades investigativas a formar: problematizar, teorizar y comprobar con un carácter de sistema, predominando los métodos participativos y entre ellos de forma particular el método investigativo.

Los medios son utilizados por profesores y estudiantes como herramientas mediadoras del proceso docente de la cátedra integradora. Deben tener relación con el objetivo planificado. Constituyen apoyo en los diferentes momentos del proceso de asimilación y ejecución de las acciones de cada habilidad investigativa a formar. La selección depende de las características del contenido y nivel de desarrollo de los estudiantes.

Las formas de organización se identifican teniendo en cuenta la flexibilidad de esta estrategia, que posibilita adecuarlas en dependencia de las necesidades del grupo.

Las clases teórico y práctica, talleres y el seminario juegan un papel fundamental, la primera realizada de una manera interactiva, donde el estudiante maneja información relacionada con el tema a tratar con antelación a la misma y donde exponen aplicaciones del tema que buscaron en otras fuentes de información y el seminario para el debate de la forma en que plantean sus problemas, las vías para darle solución y los resultados.

La evaluación se concibe con un carácter formativo, el profesor debe propiciar la autoevaluación, heteroevaluación 
y coevaluación durante la ejecución de las acciones de la tarea, mediante el trabajo individual y grupal; así como en la realización por los estudiantes del proyecto integrador al culminar la cátedra, donde demuestre como se desarrollaron las habilidades investigativas.

La evaluación de la tarea docente investigativa al culminar cada unidad de contenido posibilita la ejercitación de los estudiantes para el desarrollo del proyecto integrador al culminar la cátedra, al enfrentar al estudiante a un ejercicio similar y que pueda integrar los conocimientos científicos de la cátedra, las habilidades investigativas y la relación con la profesión para solucionar problemas científicos, con previa planificación por el profesor y su evaluación se realiza integralmente, teniendo en cuenta el desarrollo de las habilidades investigativas y la apropiación de los conocimientos de la cátedra.

Planteamiento de la situación problémica: se plantea en forma de problema, una situación relacionada con el tema abordado en el desarrollo de la cátedra integradora, contextualizado a la profesión.

Actividades a desarrollar: determinar el tipo de actividad a desarrollar en correspondencia con la situación problémica planteada y las acciones a ejecutar por los estudiantes para las habilidades investigativas problematizar, teorizar y comprobar, a formar durante el proceso docente de la cátedra.

Para el diseño de la tarea docente investigativa, se consideran como requisitos la relación con los componentes del proceso docente, el carácter problematizador e investigativo, la relación con la profesión, el vínculo teoría y práctica, la comunicación permanente y la realización de actividades individuales y colectivas. Desarrollo de la estructura de las formas de implementación, planificación de los tiempos y lugares de ejecución, así como las formas de control.

\subsection{Fase de ejecución}

El objetivo es ejecutar la tarea docente investigativa, según la planificación establecida, para la formación de las habilidades investigativas en los estudiantes de la carrera de Cultura Física. Se materializa y se implementa el diseño en la cátedra integradora.

\subsubsection{Diseño de la tarea docente investigativa en la cáte- dra integradora}

La tarea docente investigativa diseñada se realiza en función de las habilidades investigativas problematizar, teorizar y comprobar la realidad de la Cultura Física. Se instrumenta en la cátedra integradora y se orienta al estudiante desde el inicio de cada unidad de contenido. Las operaciones para la formación de estas habilidades se realizan y controlan en cada clase y al finalizar la unidad de contenido de la cátedra integradora, que culmina con la realización del proyecto integrador.
La tarea docente investigativa se desarrolla con la orientación y seguimiento paso a paso por parte del profesor, para que el estudiante ejecute y retroalimente las acciones planificadas de cada habilidad investigativa a formar.

La situación problémica planteada en la tarea, se contextualiza a la profesión y se relaciona con el conocimiento científico de la cátedra integradora. Estas situaciones implican, el estudio del contenido de la cátedra para descubrir la esencia de la situación y para resolverlas, con estos elementos de base, se diseña la tarea docente.

El diseño de esta tarea se deriva de los objetivos y de la unidad de contenido de la cátedra integradora, por ello, el número de tareas depende de esta unidad y su distribución; así como del número de horas y semanas del ciclo. Se consideran los aspectos esenciales siguientes:

- Cada una comprende el planteamiento de una situación problémica real y contextualizada a la profesión desde la cátedra.

- Diagnóstico del referencial teórico y práctico que poseen los estudiantes de los temas de la cátedra, esto permite establecer los niveles de ayuda y flexibilidad en el desarrollo de la tarea.

- La explicación que brinda la base orientadora necesaria a los estudiantes para garantizar la realización de las tareas y de las acciones.

- Control permanente, de acuerdo al periodo de tiempo establecido para cada una, de ello depende el ajuste necesario; así como carácter suficiente, variado y diferenciado de la tarea.

- Implica el empleo de las habilidades problematizar, teorizar y comprobar mediante la ejecución de sus operaciones que permiten dar respuesta a la tarea a ser evaluada.

- La tarea desarrollada al final de la unidad de contenido tributa al proyecto integrador como evaluación final de la cátedra.

Durante la ejecución, las tareas deben cumplir con las características siguientes:

- Considerar el tratamiento de los momentos funcionales de la actividad, orientación, ejecución y control.

- Propiciar la comunicación permanente que genere el debate científico, donde se planteen dudas, experiencias, puntos de vista y criterios de los participantes.

- Establecer el vínculo de la teoría con la práctica.

- Brindar niveles de ayuda mediante actividades individuales y colectivas que propicien el refuerzo del contenido y la formación de las habilidades.

- Valorar el nivel de avance en la ejecución de las acciones de cada habilidad, en las actividades tanto individual, en parejas y en pequeños grupos.

\subsection{Evaluación}

El objetivo es valorar el avance de los actores en el cumplimiento de las formas de implementación en la estrategia propuesta Conduce al seguimiento de la evolución del proceso en general y de los resultados del diseño de la tarea 
docente investigativa en la cátedra integradora, a partir del diagnóstico y de los objetivos establecidos, que permitan incluir, valorar y/o controlar cambios o modificaciones. Se realiza mediante la implementación en la cátedra integradora.

\section{Conclusiones}

Cada profesión posee particularidades que la distingue del resto y depende del contexto histórico concreto donde se desempeña, lo que resulta una necesidad el diseño de la tarea docente investigativa como componente esencial en el proceso docente actual.

La tarea docente investigativa se complementa con la búsqueda productiva del conocimiento científico, mediante el empleo de las habilidades investigativas problematizar, teorizar y comprobar la realidad de la Cultura Física desde la implementación de la tarea docente investigativa en la cátedra integradora, para tributar al desarrollo del proyecto integrador del ciclo.

Las acciones para la formación de las habilidades investigativas, permiten solucionar problemas a los estudiantes de Cultura Física, bajo la orientación del docente, vinculando las necesidades de los centros educacionales con los contenidos de la Cátedra Integradora para contribuir al trabajo de titulación y en al desempeño profesional del futuro egresado.

\section{Referencias Bibliográficas}

Aldas. (2014). La Cultura Física y su formación profesional en la República del Ecuador. EFDeportes.com, Revista Digital, 4-7.

Álvarez, C. (1996). Hacia una escuela de excelencia. La Habana: MES.
CEAACES. (2015). Modelo para la evaluación de las carreras presenciales y semi-presenciales de las universidades y escuelas politécnicas del Ecuador. Consejo de Evaluación, Acreditación y Aseguramiento de la Calidad de la Educación Superior. Quito: Gobierno.

Chirino, M. (2002). Perfeccionamiento de la formación inicial investigativa de los profesionales de la educación (Inf. Téc.). La Habana.

Chirino, M. (2003). La formación inicial investigativa en los ISP. Sistema de alternativas metodológicas. Premio de ciencia e innovación educativa (Inf. Téc.). La Habana.

Chirino, M. (2005). El trabajo independiente desde una concepción desarrolladora del proceso deenseñanza aprendizaje. La Habana: Pueblo y Educación.

Larrea, E. (2013). El Currículo de la Educación Superior desde la Complejidad Sistémica (Inf. Téc.). Quito.

Sánchez, L., y et Al. (2010). Tareas docentes investigativas en el perfil Servicios Farmacéuticos en Cienfuegos. Medisur, 31.

UCACUE. (2016). Manual de Investigación Formativa. Ucacue: Formato Digital.

Recibido: 18 de septiembre de 2019

Aceptado: 25 de enero de 2020 